\title{
Equivalence of the (Generalised) Hadamard and Microlocal Spectrum Condition for (Generalised) Free Fields in Curved Spacetime
}

\author{
Ko Sanders ${ }^{1,2}$ \\ 1 Institute of Theoretical Physics, University of Göttingen, Friedrich-Hund-Platz 1, \\ D-37077 Göttingen, Germany. E-mail: jacobus.sanders@theorie.physik.uni-goe.de \\ 2 Courant Research Centre, "Higher Order Structures in Mathematics", \\ University of Göttingen, Göttingen, Germany
}

Received: 5 May 2009 / Accepted: 9 June 2009

Published online: 13 August 2009 - (C) The Author(s) 2009. This article is published with open access at Springerlink.com

\begin{abstract}
We prove that the singularity structure of all $n$-point distributions of a state of a generalised real free scalar field in curved spacetime can be estimated if the twopoint distribution is of Hadamard form. In particular this applies to the free field and the result has applications in perturbative quantum field theory, showing that the class of all Hadamard states is the state space of interest. In our proof we assume that the field is a generalised free field, i.e. that it satisfies scalar (c-number) commutation relations, but it need not satisfy an equation of motion. The same arguments also work for anti-commutation relations and for vector-valued fields. To indicate the strengths and limitations of our assumption we also prove the analogues of a theorem by Borchers and Zimmermann on the self-adjointness of field operators and of a weak form of the Jost-Schroer theorem. The original proofs of these results make use of analytic continuation arguments. In our case no analyticity is assumed, but to some extent the scalar commutation relations can take its place.
\end{abstract}

\section{Introduction}

The study of quantum field theories in curved spacetime is simplified considerably by the use of techniques from microlocal analysis to study the singularities of $n$-point distributions. Ever since Radzikowski [16] has shown that Hadamard states of the real free scalar field can be characterised by the wave front set of their two-point distributions, these techniques have been on the increase as a suitable replacement of the Fourier transform in Minkowski spacetime. This enabled Brunetti, Fredenhagen and Köhler [2] to introduce a microlocal spectrum condition ( $\mu \mathrm{SC})$ for general real scalar fields, which is a (smoothly) generally covariant condition that generalises Wightman's spectrum condition.

The generalisation is only possible at a price: whereas the $n$-point distributions of a Wightman field are the boundary values of analytic functions, this is no longer so in curved spacetimes. In [21] an analytic microlocal spectrum condition (A $\mu \mathrm{SC})$ was introduced on analytic spacetimes in order to provide an amount of analyticity analogous 
to the Wightman case, but the requirement that the metric be analytic in some analytic structure on the manifold, although technically advantageous, seems to be unphysically restrictive. In fact, a generic curved spacetime cannot be expected to be analytic at all, so all arguments involving analytic continuation have to be reexamined in the context of quantum field theory in curved spacetime.

In this work we will not require any analyticity, but instead we consider a real scalar field which satisfies scalar (i.e. c-number) commutation relations ${ }^{1}$. These fields, which include the real free scalar field, will be called generalised free fields, following the terminology for the Wightman framework in Minkowski spacetime (see e.g. [10]), although in curved spacetime not much seems to be known about them. As our main result we will prove that an estimate on the singularities of the two-point distribution ("generalised Hadamard condition") implies estimates on the singularities of all $n$-point distributions. In particular, all truncated $n$-point distributions with $n \neq 2$ will be shown to be smooth and consequently the state will satisfy the $\mu \mathrm{SC}$. An easy application is that the class of generalised Hadamard states is closed under operations from the algebra of observables. Moreover, all Hadamard states of a free field can be extended to the extended algebra of Wick polynomials and time-ordered products as constructed by Hollands and Wald $[7,8]$.

After that we will investigate the strength of our assumption by proving the analogues of a result by Borchers and Zimmermann [1] on the self-adjointness of field operators and a very weak version of the Jost-Schroer theorem $[4,11,15]$. In both cases the original proofs rely on analytic continuation arguments, but in our case no analyticity is assumed. The commutation relations take the place of analyticity to a certain extent, but not fully, and we have had to weaken the conclusion of the Jost-Schroer theorem accordingly.

The organisation of our paper is as follows: we first establish our notation for quantum field theory in curved spacetime in Sect. 2. There we also present the $\mu \mathrm{SC}$, the (generalised) Hadamard condition and the truncated $n$-point distributions and we collect some results concerning the singularities of the two-point distribution. In Sect. 3 we introduce the commutation relations and give two equivalent characterisations of generalised free fields. Section 4 contains our main results concerning the singularity structure of higher $n$-point distributions and truncated $n$-point distributions, as well as a result on the comparison of $n$-point distributions of different states. In Sect. 5 we discuss the generalisations of the result by Borchers and Zimmermann and the Jost-Schroer theorem. We conclude with some easy applications and an outlook in Sect.6. For an introduction to microlocal analysis we refer to Chap. 8 of [9].

\section{Real Scalar Quantum Fields and the Microlocal Spectrum Condition}

Let $M=(\mathcal{M}, g)$ be a spacetime, i.e. $\mathcal{M}$ is a smooth, connected manifold of dimension $D \geq 2$ with the smooth Lorentzian metric $g$, where we use the signature convention $+-\cdots-$. We let $V \subset T M$ denote the set of all causal tangent vectors (including 0-vectors) and we let $V^{*} \subset T^{*} M$ be its dual, i.e. the image of $V$ under the identification of $T M$ with $T^{*} M$ via the metric. We assume that $M$ is time-oriented, so we can define the future and past causal cones $V^{ \pm} \subset T M$ and their duals, $V^{* \pm} \subset T^{*} M$. We use $\mathcal{Z}$ to denote the zero section of a vector bundle (it will always be clear from the context which vector bundle is meant).

\footnotetext{
1 Our results also work for anti-commutation relations and for vector-valued fields.
} 
A real scalar quantum field on the spacetime $M$ can be described using the BorchersUhlmann algebra. Here we adopt the convention that the space $M^{\times 0}$ consists of a single point, so that $C_{0}^{\infty}\left(M^{\times 0}\right)=\mathbb{C}$.

Definition 2.1. The (scalar) Borchers-Uhlmann algebra on the spacetime $M$ is defined to be the topological ${ }^{*}$-algebra $\mathcal{U}_{M}:=\oplus_{n=0}^{\infty} C_{0}^{\infty}\left(M^{\times n}\right)$, where we allow only finite direct sums and where

1. the product is determined by the linear extension of

$$
f\left(x_{n+m}, \ldots, x_{n+1}\right) g\left(x_{n}, \ldots, x_{1}\right):=(f \otimes g)\left(x_{n+m}, \ldots, x_{1}\right),
$$

2. the ${ }^{*}$-operation is determined by anti-linear extension of

$f^{*}\left(x_{n}, \ldots, x_{1}\right):=\overline{f\left(x_{1}, \ldots, x_{n}\right)}$,

3. as a topological space $\mathcal{U}_{M}$ is the strict inductive limit

$$
\mathcal{U}_{M}=\cup_{N=0}^{\infty} \oplus_{n=0}^{N} C_{0}^{\infty}\left(K_{N}^{\times n}\right),
$$

where $K_{N}$ is an exhausting (and increasing) sequence of compact subsets of $\mathcal{M}$ and each $C_{0}^{\infty}\left(K_{N}^{\times n}\right)$ is given the test-function topology (cf. [19] Theorem 2.6.4).

A state on the Borchers-Uhlmann algebra is a normalised continuous positive linear $\operatorname{map} \omega: \mathcal{U}_{M} \rightarrow \mathbb{C}$.

The topology of $\mathcal{U}_{M}$ is such that $f_{j}=\oplus_{n} f_{j}^{(n)}$ converges to $f=\oplus_{n} f^{(n)}$ if and only if for all $n$ we have $f_{j}^{(n)} \rightarrow f^{(n)}$ in $C_{0}^{\infty}\left(M^{\times n}\right)$ and all $f_{j}^{(n)}$ vanish if $n \geq N$ for some $N>0$. A state therefore consists of a sequence of $n$-point distributions, $\omega=\left\{\omega_{n}\right\}_{n=0}^{\infty}$, where $\omega_{n}$ is a distribution on $M^{\times n}$. The algebra $\mathcal{U}_{M}$ has the unit $I=1 \oplus 0 \oplus 0 \ldots$ and the normalisation of the state $\omega$ means that $\omega(I)=\omega_{0}=1$. Given a state one can construct the GNS-representation $\pi_{\omega}$ on a Hilbert space $\mathcal{H}_{\omega}$ with a dense domain $\mathcal{D}_{\omega}$ that contains a vector $\Omega_{\omega}$ such that: $\mathcal{D}_{\omega}=\pi_{\omega}\left(\mathcal{U}_{M}\right) \Omega_{\omega}$ and $\omega(A)=\left\langle\Omega_{\omega}, \pi_{\omega}(A) \Omega_{\omega}\right\rangle$ for each $A \in \mathcal{U}_{M}$. The GNS-quadruple $\left(\pi_{\omega}, \mathcal{H}_{\omega}, \mathcal{D}_{\omega}, \Omega_{\omega}\right)$ is the unique quadruple with these properties, up to unitary equivalence (see [20] Theorem 8.6.2).

Instead of the $n$-point distributions one often considers the truncated $n$-point distributions of a state $\omega$, which we will now define. For $n \geq 1$ we let $\mathcal{P}_{n}$ denote the set of all partitions of the set $\{1, \ldots, n\}$ into pairwise disjoint subsets, which are ordered from low to high. If $r$ is an ordered set in the partition $P \in \mathcal{P}_{n}$ we write $r \in P$ and we denote the elements of $r$ by $r(1)<\cdots<r(|r|)$, where $|r|$ is the number of elements in $r$. The truncated $n$-point distributions $\omega_{n}^{T}, n \geq 1$, of a state $\omega$ are defined implicitly in terms of the $n$-point distributions $\omega_{n}$ by:

$$
\omega_{n}\left(x_{n}, \ldots, x_{1}\right)=\sum_{P \in \mathcal{P}_{n}} \prod_{r \in P} \omega_{|r|}^{T}\left(x_{r(|r|)}, \ldots, x_{r(1)}\right) .
$$

Note that this equation can be solved iteratively for $\omega_{n}^{T}$ order by order.

Definition 2.2. A state $\omega$ is called quasi-free if and only if $\omega_{n}^{T} \equiv 0$ for all $n \neq 2$.

We will denote by $\Phi$ the canonical injection $C_{0}^{\infty}(M) \subset \mathcal{U}_{M}$, which sends $f$ to

$$
\Phi(f):=0 \oplus f \oplus 0 \oplus \ldots
$$

The map $\Phi$ is a distribution with values in $\mathcal{U}_{M}$ and it represents the real scalar quantum field. In the GNS-representation of a state $\omega$ the field is represented by $\Phi^{\omega}(f):=$ $\pi_{\omega}(\Phi(f))$. For our current purposes it is convenient not to impose commutation 
relations, causality or an equation of motion on the field $\Phi$, but to let them be dictated for $\Phi^{\omega}$ by the state. This will be done in Sect. 3 .

We now give an equivalent reformulation of the $\mu \mathrm{SC}$ of Brunetti, Fredenhagen and Köhler [2], starting with the introduction of some terminology.

Definition 2.3. We let $\mathcal{G}_{n}$ denote the set of all graphs with $n$ vertices and finitely many edges. An immersion of a graph $G \in \mathcal{G}_{n}$ into the spacetime $M$ consists of an assignment of

1. a point $x(i) \in M$ to each vertex $v_{i}$ of $G$,

2. a piecewise smooth curve $\gamma_{r}$ between $x(i)$ and $x(j)$ to every edge $e_{r}$ of $G$ that connects $v_{i}$ and $v_{j}$,

3. a causal, future pointing covector field $\xi_{r}$ on $\gamma_{r}$ to each $e_{r}$, so that $\xi_{r}$ is covariantly constant, $\nabla \xi_{r}=0$, along $\gamma_{r}$.

An immersion of a graph $G \in \mathcal{G}_{n}$ into the spacetime $M$ is called causal, resp. lightlike, iff the curves $\gamma_{r}$ are causal, resp. light-like.

We say that a point $\left(x_{n}, k_{n} ; \ldots ; x_{1}, k_{1}\right) \in T^{*} M^{\times n} \backslash \mathcal{Z}$ is instantiated by an immersion of a graph $G \in \mathcal{G}_{n}$ if and only if for each $i=1, \ldots, n$ the immersion sends the vertex $v_{i}$ to $x_{i}$ and

$$
k_{i}=\sum_{e_{r} \text { between } i \text { and } j>i} \xi_{r}\left(x_{i}\right)-\sum_{e_{r} \text { between } j<i \text { and } i} \xi_{r}\left(x_{i}\right) .
$$

Recall that $\mathcal{Z}$ denotes the zero section of a vector bundle. The covector field $\xi_{r}$ is to be thought of as a singularity, propagating along the curve $\gamma_{r}$ from $x(i)$ to $x(j)$. The following sets describe the singularities that we allow the $n$-point distributions to have:

$$
\begin{aligned}
\Gamma_{n}:= & \left\{\left(x_{n}, k_{n} ; \ldots ; x_{1}, k_{1}\right) \in T^{*} M^{\times n} \backslash \mathcal{Z} \mid \exists G \in \mathcal{G}_{n} \text { and an immersion of } G\right. \\
& \text { into } \left.M \text { which instantiates the point }\left(x_{n}, k_{n} ; \ldots ; x_{1}, k_{1}\right)\right\} .
\end{aligned}
$$

The sets $\Gamma_{n}^{c}$, resp. $\Gamma_{n}^{l l}$, are defined similarly, but using only causal, resp. light-like, immersions of graphs. In general we will write $\Gamma_{n}^{\bullet}$, where ${ }^{\bullet}$ denotes either no superscript, or $c$ or $l l$.

Definition 2.4. A state $\omega$ satisfies the microlocal spectrum condition ( $\mu S C)$ with smooth, resp. causal, resp. light-like immersions, iff for all $n \in \mathbb{N}$ we have $W F\left(\omega_{n}\right) \subset$ $\Gamma_{n}^{\bullet}$, where $\bullet$ denotes no superscript, resp. c, resp. $l l$.

If $M$ is an analytic spacetime then $\omega$ satisfies the analytic microlocal spectrum condition $(A \mu S C)$ with smooth, resp. causal, resp. light-like immersions, iff for all $n \in \mathbb{N}$

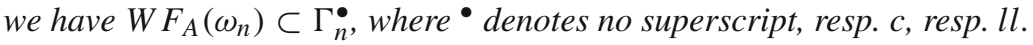

The usefulness of these restrictions on the singularities of the $n$-point distributions derives largely from the properties of the sets $\Gamma_{n}^{\bullet}$ :

Proposition 2.5. The sets $\Gamma_{n}^{\bullet}$, with a fixed choice for the superscript ${ }^{\bullet}$, have the following properties:

1. each $\Gamma_{n}^{\bullet} \subset T^{*} M^{\times n} \backslash \mathcal{Z}$ is a convex cone,

2. $\Gamma_{n}^{\bullet} \cap-\Gamma_{n}^{\bullet}=\emptyset$,

3. for every partition $P \in \mathcal{P}_{n}$ with ordered subsets $r_{1}, \ldots, r_{m}$ we have $\pi\left(\left(\Gamma_{\left|r_{1}\right|}^{\bullet} \cup \mathcal{Z}\right) \times \ldots \times\left(\Gamma_{\left|r_{m}\right|}^{\bullet} \cup \mathcal{Z}\right)\right) \subset \Gamma_{n}^{\bullet} \cup \mathcal{Z}$, where the permutation $\pi$ acts on the indices and sends $\left(r_{1}, \ldots, r_{m}\right)$ to $\{1, \ldots, n\}$. 


\section{4. $\left(x_{1}, k_{1} ; \ldots ; x_{n}, k_{n}\right) \in-\Gamma_{n}^{\bullet}$ iff $\left(x_{n}, k_{n} ; \ldots ; x_{1}, k_{1}\right) \in \Gamma_{n}^{\bullet}$.}

Proof. We refer to [2] Lemma 4.2 for a proof of the first property. The second property follows from the first and the third property follows immediately from the definitions, using the unions of disconnected instantiating graphs (cf. [2] Prop.4.3). The fourth property follows directly from the definitions.

Lemma 2.6. A state $\omega$ satisfies the $\mu S C$ with smooth, resp. causal, resp. light-like immersions iff $W F\left(\omega_{n}^{T}\right) \subset \Gamma_{n}^{\bullet}$ for all $n \in \mathbb{N}$, where ${ }^{\bullet}$ denotes no superscript, resp. c, resp. $l l$. The same result holds in the analytic case.

Proof. We prove by induction on $n \in \mathbb{N}$ that $W F\left(\omega_{n}^{T}\right) \subset \Gamma_{n}^{\bullet}$ if and only if $W F\left(\omega_{n}\right) \subset$ $\Gamma_{n}^{\bullet}$. For $n=1$ this holds because $\omega_{1}^{T}=\omega_{1}$. Now assume that the claim holds for $i=1, \ldots, n-1$ for some $n \geq 2$. From Eq. (1) we see that $\omega_{n}-\omega_{n}^{T}$ can be expressed as a sum, whose wave front set is contained in $\Gamma_{n}^{\bullet}$ by Items 1 and 3 in Proposition 2.5. Using Item 1 of this proposition once more we see that $W F\left(\omega_{n}\right) \subset \Gamma_{n}^{\bullet}$ if and only if $W F\left(\omega_{n}^{T}\right) \subset \Gamma_{n}^{\bullet}$. The argument is purely combinatorical, so it remains true in the analytic case.

A much weaker condition than the $\mu \mathrm{SC}$ is the Hadamard condition. This condition only places a restriction on the singularities of the two-point distribution so as to enable the renormalisation of the stress-energy-momentum tensor of a free scalar field (see [23]). Because our field need not be free we will consider the following immediate generalisation of the Hadamard condition:

Definition 2.7. A state $\omega$ is called a generalised Hadamard state iff $W F\left(\omega_{2}\right) \subset \Gamma_{2}$.

Note that a (generalised) Hadamard state need not be quasi-free. We will show in Sect. 3 that the generalised Hadamard condition reduces to the Hadamard condition in the case of free fields.

To complete this section we will now collect some small but useful results on the relation between the generalised Hadamard condition and the two-point distribution. For this purpose we define the symmetric and anti-symmetric part of the two-point distribution by

$$
\omega_{2 \pm}\left(x_{2}, x_{1}\right):=\frac{1}{2}\left(\omega_{2}\left(x_{2}, x_{1}\right) \pm \omega_{2}\left(x_{1}, x_{2}\right)\right)
$$

Proposition 2.8. If $\omega$ is a generalised Hadamard state, then we have:

1. $\left(x_{2}, k_{2} ; x_{1}, k_{1}\right) \in W F\left(\omega_{2 \pm}\right)$ iff $\left(x_{1}, k_{1} ; x_{2}, k_{2}\right) \in W F\left(\omega_{2 \pm}\right)$ iff $\left(x_{2},-k_{2} ; x_{1},-k_{1}\right) \in W F\left(\omega_{2 \pm}\right)$,

2. $W F\left(\omega_{2+}\right)=W F\left(\omega_{2-}\right) \subset \Gamma_{2} \cup-\Gamma_{2}$,

3. $W F\left(\omega_{2}\right)=W F\left(\omega_{2-}\right) \cap \Gamma_{2}$.

Proof. The idea of this proof is borrowed from Proposition 6.1 in [21]. The positivity of $\omega$ implies that $\overline{\omega_{2}}\left(x_{2}, x_{1}\right)=\omega_{2}\left(x_{1}, x_{2}\right)$, so from Eq. (3) we find that $\overline{\omega_{2 \pm}}\left(x_{2}, x_{1}\right)=$ $\omega_{2 \pm}\left(x_{1}, x_{2}\right)= \pm \omega_{2 \pm}\left(x_{2}, x_{1}\right)$, from which the first item follows. That $W F\left(\omega_{2 \pm}\right) \subset$ $\Gamma_{2} \cup-\Gamma_{2}$ for a generalised Hadamard state is clear from the definition. Now suppose that $\left(x_{2}, k_{2} ; x_{1}, k_{1}\right) \in W F\left(\omega_{2 \pm}\right)$. Then we can distinguish two cases, namely either $\left(x_{2}, k_{2} ; x_{1}, k_{1}\right) \notin W F\left(\omega_{2}\right)$ or $\left(x_{2},-k_{2} ; x_{1},-k_{1}\right) \notin W F\left(\omega_{2}\right)$ by Eq. (3) and Statement 2 of Proposition 2.5. Using $\omega_{2 \pm}=\omega_{2}-\omega_{2 \mp}$ and the properties under the first item 
we find that either $\left(x_{2}, k_{2} ; x_{1}, k_{1}\right) \in W F\left(\omega_{2 \mp}\right)$ or $\left(x_{2},-k_{2} ; x_{1},-k_{1}\right) \in W F\left(\omega_{2 \mp}\right)$, as the case may be, and hence that $\left(x_{2}, k_{2} ; x_{1}, k_{1}\right) \in W F\left(\omega_{2 \mp}\right)$. Thus $W F\left(\omega_{2+}\right) \subset$ $W F\left(\omega_{2-}\right)$ and the opposite inclusion can be proved in the same way, which proves the second item. For the last item we use again the definition $2 \omega_{2-}=\omega_{2}-\tilde{\omega}_{2}$, where $\tilde{\omega}_{2}\left(x_{2}, x_{1}\right):=\omega_{2}\left(x_{1}, x_{2}\right)$. By the assumption on $\omega_{2}$ we have $W F\left(\omega_{2}\right) \cap W F\left(\tilde{\omega}_{2}\right)=\emptyset$. Hence we deduce: $W F\left(\omega_{2}\right) \subset W F\left(\omega_{2-}\right), W F\left(\tilde{\omega}_{2}\right) \subset W F\left(\omega_{2-}\right)$ and $W F\left(\omega_{2-}\right) \subset$ $W F\left(\omega_{2}\right) \cup W F\left(\tilde{\omega}_{2}\right)$, from which it follows that $W F\left(\omega_{2-}\right)=W F\left(\omega_{2}\right) \cup W F\left(\tilde{\omega}_{2}\right)$. Intersecting with $\Gamma_{2}$ then gives the result.

The following result on the comparison of two generalised Hadamard states is well known and lies at the basis of the renormalisation of the stress-energy-momentum tensor in the free field case:

Lemma 2.9. For two generalised Hadamard states $\omega, \omega^{\prime}$ we have that $\omega_{2}-\omega_{2}^{\prime}$ is smooth iff $\omega_{2-}-\omega_{2-}^{\prime}$ is smooth.

Proof. We define $w\left(x_{2}, x_{1}\right):=\left(\omega_{2}-\omega_{2}^{\prime}\right)\left(x_{2}, x_{1}\right), \tilde{w}\left(x_{2}, x_{1}\right):=w\left(x_{1}, x_{2}\right)$ and $w_{2-}:=$ $\frac{1}{2}(w-\tilde{w})$ and argue as in the proof of Proposition 2.8: $W F(w) \subset \Gamma_{2}, W F(\tilde{w}) \subset$ $-\Gamma_{2}$, and hence $W F(w) \cap W F(\tilde{w})=\emptyset$. It then follows from $w-\tilde{w}=2 w_{2-}$ that $W F\left(w_{2-}\right)=W F(w) \cup W F(\tilde{w})$. Now $W F\left(w_{2-}\right)=\emptyset$ if and only if $W F(w)=\emptyset$, which proves the statement.

\section{Generalised Free Fields in Curved Spacetime}

In this section we define a number of physical properties that the state $\omega$ may satisfy and derive some easy results concerning them:

Definition 3.1. The state $\omega$ is called causal iff $\omega$ descends to a state on $\mathcal{U}_{M} / J$, where $J \subset \mathcal{U}_{M}$ is the *-ideal generated by all elements of the form $f \otimes h-h \otimes f$ where the supports of $f, h \in C_{0}^{\infty}(M)$ are causally disjoint.

A state $\omega$ satisfies the Klein-Gordon equation with mass $m$ and scalar curvature coupling $\xi$ iff $\omega$ descends to a state on $\mathcal{U}_{M} / J$, where $J \subset \mathcal{U}_{M}$ is the ${ }^{*}$-ideal generated by all elements of the form $\left(\square+m^{2}+\xi R\right) f$, where $\square$ is the d'Alembertian and $R$ the scalar curvature.

Given a bi-distribution $E$ on $M^{\times 2}$ we say that the state $\omega$ is a generalised free field state with commutator $E$ iff $\omega$ satisfies the commutation relations with commutator $E$, i.e. iff $\omega$ descends to a state on $\mathcal{U}_{M} / J$, where $J \subset \mathcal{U}_{M}$ is the ${ }^{*}$-ideal generated by all elements of the form $f \otimes h-h \otimes f-i E(f, h) I$.

A generalised free field state $\omega$ on a globally hyperbolic spacetime is called a free field state iff it satisfies the Klein-Gordon equation with mass $m$ and scalar curvature coupling $\xi$ and $E=E_{m, \xi}$, the difference of the advanced and retarded fundamental solutions of the Klein-Gordon equation $\left(\square+m^{2}+\xi R\right) \phi=0$.

The first three properties above can be written equivalently in terms of the represented field as:

$$
\begin{aligned}
{\left[\Phi^{\omega}(f), \Phi^{\omega}(h)\right] } & =0, \quad \text { supp } f \cap J(\operatorname{supp} h)=\emptyset, \\
\left(\square+m^{2}+\xi R\right) \Phi^{\omega} & =0, \\
{\left[\Phi^{\omega}(f), \Phi^{\omega}(h)\right] } & =i E(f, h) I .
\end{aligned}
$$


We have chosen to allow very general distributions $E$ to appear in the commutation relations in order to emphasise that their precise form does not matter for our arguments. In particular, our commutation relations need not imply causality and our arguments also hold for anti-commutation relations. However, it is important that the commutator of two smeared field operators is a scalar. Note that $E$ must be anti-symmetric, $E(f, h)=-E(h, f)$ for all $f, h \in C_{0}^{\infty}(M)$, for the commutation relations to make sense.

Instead of the free field commutator $E=E_{m, \xi}$ one can take for example $E\left(x_{2}, x_{1}\right):=$ $\int_{0}^{\infty} \int E_{m, \xi}\left(x_{2}, x_{1}\right) f(m, \xi) d \xi d m$, where $f$ is a compactly supported smooth function. In fact, in the Wightman framework in Minkowski spacetimeone can use the KällenLehmann representation of the two-point distribution to prove that $E$ must be of this form for a suitable distribution $f(m) \delta(\xi)$. (We can take $\xi=0$ because $R \equiv 0$ in Minkowski spacetime.) Whether such a result still holds in curved spacetime is not clear, because no suitable replacement of the Källen-Lehmann is currently available. We leave these issues for a future investigation.

It is worthwhile to note the following:

Proposition 3.2. If $\omega$ is a generalised Hadamard state and a generalised free field state with commutator $E$, then $E=-2 i \omega_{2-}, W F(E) \subset \Gamma_{2} \cup-\Gamma_{2}$ and $W F\left(\omega_{2}\right)=$ $W F(E) \cap \Gamma_{2}$.

Proof. The first equality follows by applying $\omega$ to the commutation relations (6). The others follow from the last two items of Proposition 2.8.

Note that $W F\left(E_{m, \xi}\right) \subset \Gamma_{2}^{l l} \cup-\Gamma_{2}^{l l}$, so in this case we must have $W F\left(\omega_{2}\right) \subset \Gamma_{2}^{l l}$.

Corollary 3.3. If a state $\omega$ satisfies the Klein-Gordon equation with parameters $m, \xi$ on a globally hyperbolic spacetime $M$ and the commutation relations with commutator $E_{m, \xi}$, then it is a generalised Hadamard state if and only if it is a Hadamard state.

Proof. The result of Radzikowski implies that a free field state on a globally hyperbolic spacetime which satisfies the commutation relations with commutator $E_{m, \xi}$ is a Hadamard state if and only if $W F\left(\omega_{2}\right)=W F\left(E_{m, \xi}\right) \cap \Gamma_{2}$. The result therefore follows from Proposition 3.2.

We see from Proposition 3.2 and Lemma 2.9 that for two generalised free field states $\omega$ and $\omega^{\prime}$ with commutator functions $E$ and $E^{\prime}$ respectively, $\omega_{2}-\omega_{2}^{\prime}$ is smooth iff $E-E^{\prime}$ is smooth. In general, however, even $E_{m, \xi}-E_{m^{\prime}, \xi^{\prime}}$ will not be smooth, even though both have the same wave front sets. Indeed, if $E_{m, \xi}-E_{m^{\prime}, \xi^{\prime}}$ is smooth and if we define $K_{x_{2}}:=\square+m^{2}+\xi R$ acting on the variable $x_{2}$ and similarly for $K^{\prime}$, then the following is also smooth:

$$
\begin{aligned}
& K_{x_{2}}^{\prime}\left(E_{m, \xi}-E_{m^{\prime}, \xi^{\prime}}\right)\left(x_{2}, x_{1}\right)=K_{x_{2}}^{\prime} E_{m, \xi}\left(x_{2}, x_{1}\right) \\
& \quad=\left(K_{x_{2}}^{\prime}-K_{x_{2}}\right) E_{m, \xi}\left(x_{2}, x_{1}\right) \\
& =\left(\left(m^{\prime}\right)^{2}-m^{2}+\xi^{\prime} R\left(x_{2}\right)-\xi R\left(x_{2}\right)\right) E_{m, \xi}\left(x_{2}, x_{1}\right) .
\end{aligned}
$$

Because $E_{m, \xi}\left(x_{2}, x_{1}\right)$ is singular whenever $x_{1}$ and $x_{2}$ can be connected by a light-like geodesic, we would then have to have $\left(m^{\prime}\right)^{2}-m^{2}+\xi^{\prime} R\left(x_{2}\right)-\xi R\left(x_{2}\right) \equiv 0$. In general, however, this is not the case.

We conclude this section by proving a useful equivalent characterisation of generalised free fields in terms of the truncated $n$-point distributions (see note added in proof): 
Proposition 3.4. A state $\omega$ is a generalised free field state iff all the truncated n-point distributions $\omega_{n}^{T}$ with $n \neq 2$ are symmetric in their arguments.

In the case where we have anti-commutation relations instead of commutation relations a similar proof shows that the truncated $n$-point distributions are anti-symmetric for $n \neq 2$.

Proof. First assume that $\omega_{n}^{T}$ is symmetric for all $n \neq 2$. For $n \geq 2$ we then use Eq. (1) to see that for any $1 \leq i<n$,

$$
\begin{aligned}
& \omega_{n}\left(x_{n}, \ldots, x_{1}\right)-\omega_{n}\left(x_{n}, \ldots, x_{i}, x_{i+1}, \ldots, x_{1}\right) \\
& \quad=2 \omega_{2-}\left(x_{i+1}, x_{i}\right) \omega_{n-2}\left(x_{n}, \ldots, \hat{x}_{i+1}, \hat{x}_{i}, \ldots, x_{1}\right) .
\end{aligned}
$$

Here we noted that most terms cancel out, either by the hypothesis or by the fact that $i$ and $i+1$ are subsequent indices. The remaining terms have been collected together using once again Eq. (1). By definition this equation means that $\omega$ satisfies the commutation relations with commutator $E=-2 i \omega_{2-}$.

For the opposite direction we assume that $\omega$ satisfies the commutation relations (necessarily with $E=-2 i \omega_{2-}$ ). We use similar arguments as above to prove by induction that $\omega_{n}^{T}$ is symmetric for $n \geq 3$ (for $n=1,2$ there is nothing to prove):

$$
\begin{aligned}
& \omega_{3}^{T}\left(x_{3}, x_{2}, x_{1}\right)-\omega_{3}^{T}\left(x_{2}, x_{3}, x_{1}\right) \\
& \quad=\omega_{3}\left(x_{3}, x_{2}, x_{1}\right)-\omega_{3}\left(x_{2}, x_{3}, x_{1}\right)-\left(\omega_{2}^{T}\left(x_{3}, x_{2}\right)-\omega_{2}^{T}\left(x_{2}, x_{3}\right)\right) \omega_{1}^{T}\left(x_{1}\right) \\
& \quad=2 \omega_{2-}\left(x_{3}, x_{2}\right) \omega_{1}\left(x_{1}\right)-2 \omega_{2-}\left(x_{3}, x_{2}\right) \omega_{1}\left(x_{1}\right)=0 .
\end{aligned}
$$

A similar result holds for the transposition in the indices 1 and 2, from which the invariance under all permutations follows for $n=3$. Next we consider $n>3$ and assume that the claim holds for all $\omega_{n^{\prime}}^{T}$ with $0 \leq n^{\prime} \leq n-1$. Again it suffices to prove that $\omega_{n}^{T}\left(x_{n}, \ldots, x_{1}\right)$ is invariant under a transposition of the indices $i$ and $i+1$ for some $1 \leq i \leq n-1$, because such transpositions generate the group of all permutations. Using the induction hypothesis we find similarly:

$$
\begin{aligned}
& \omega_{n}^{T}\left(x_{n}, \ldots, x_{1}\right)-\omega_{n}^{T}\left(x_{n}, \ldots, x_{i}, x_{i+1}, \ldots, x_{1}\right) \\
& \quad=\omega_{n}\left(x_{n}, \ldots, x_{1}\right)-\omega_{n}\left(x_{n}, \ldots, x_{i}, x_{i+1}, \ldots, x_{1}\right) \\
& \quad-\left(\omega_{2}^{T}\left(x_{i+1}, x_{i}\right)-\omega_{2}^{T}\left(x_{i}, x_{i+1}\right)\right) \omega_{n-2}\left(x_{n}, \ldots, \hat{x}_{i+1}, \hat{x}_{i}, \ldots, x_{1}\right)=0 .
\end{aligned}
$$

This completes the proof.

The previous proposition is reminiscent of, but certainly not equivalent to, the result in [10] that a vacuum state $\omega$ of a Wightman field theory is causal if and only if the $n$-point distributions $\omega_{n}$, extended to suitable complex domains, are symmetric in their arguments in those domains. That result, however, uses the Bargmann-Hall-Wightman theorem, whereas our result relies solely on elementary combinatorics (cf. [10] Sect. 4.4, $[6,11])$.

Finally we note the following corollary of Proposition $3.4^{2}$ :

Corollary 3.5. A quasi-free state satisfies the commutation relations with commutator $E=-2 i \omega_{2-}$.

Proof. By Definition 2.2 of a quasi-free state $\omega_{n}^{T}$ is symmetric for $n \neq 2$.

\footnotetext{
${ }^{2}$ We thank Prof. Rehren for pointing this out to us at an early stage.
} 


\section{Equivalence of the Hamadard and Microlocal Spectrum Conditions}

We now start our analysis of the singularities of higher $n$-point distributions of a generalised free field state with a result that exploits the positivity of the state.

Proposition 4.1. Let $\omega$ be a generalised Hadamard state and assume that for $n \geq 1$ we have $\left(x_{n}, k_{n} ; \ldots ; x_{1}, k_{1}\right) \in W F\left(\omega_{n}\right)$. Then $\left(x_{1}, k_{1}\right) \in V^{*+} \cup \mathcal{Z}$ and $\left(x_{n}, k_{n}\right) \in V^{*-} \cup \mathcal{Z}$. In particular, $W F\left(\omega_{1}\right)=\emptyset$.

Proof. The positivity of $\omega$ implies $\overline{\omega_{n}\left(f_{n}, \ldots, f_{1}\right)}=\omega_{n}\left(\bar{f}_{1}, \ldots, \bar{f}_{n}\right)$, and hence the second statement follows from the first. In fact, the positivity allows us to perform the GNSconstruction, which yields a representation $\pi_{\omega}$ of $\mathcal{U}_{M}$ on a Hilbert space $\mathcal{H}_{\omega}$ by closable operators and a vector $\Omega_{\omega} \in \mathcal{H}_{\omega}$ such that $\omega(A)=\left\langle\Omega_{\omega}, \pi_{\omega}(A) \Omega_{\omega}\right\rangle$ for all $A \in \mathcal{U}_{M}$. We can then define the $\mathcal{H}_{\omega}$-valued distributions $\phi_{m}\left(f_{m}, \ldots, f_{1}\right):=\pi_{\omega}\left(f_{m} \otimes \ldots \otimes f_{1}\right) \Omega_{\omega}$ for all $m \in \mathbb{N}$. Using the inner product of $\mathcal{H}_{\omega}$ we can write:

$$
\begin{aligned}
\omega_{n}\left(f_{n}, \ldots, f_{1}\right) & =\left\langle\phi_{n-1}\left(\bar{f}_{2}, \ldots, \bar{f}_{n}\right), \phi_{1}\left(f_{1}\right)\right\rangle, \\
\omega_{2}\left(f_{2}, f_{1}\right) & =\left\langle\phi_{1}\left(\bar{f}_{2}\right), \phi_{1}\left(f_{1}\right)\right\rangle .
\end{aligned}
$$

The calculus of Hilbert space-valued distributions (see e.g. [21] Proposition 2.2 or [18] Theorem A.1.6) now means that $\left(x_{n}, k_{n} ; \ldots ; x_{1}, k_{1}\right) \in W F\left(\omega_{n}\right)$ implies $\left(x_{1}, k_{1}\right) \in$ $W F\left(\phi_{1}\right) \cup \mathcal{Z}$ and if $k_{1} \neq 0$ then $\left(x_{1},-k_{1} ; x_{1}, k_{1}\right) \in W F\left(\omega_{2}\right)$. The conclusion follows from the assumption that $W F\left(\omega_{2}\right) \subset \Gamma_{2}$.

Proposition 4.1 has some nice consequences in the case of generalised free fields:

Theorem 4.2. Let $\omega$ be a generalised Hadamard state which is also a generalised free field state. Then $\omega_{2}^{T}-\omega_{2}$ and $\omega_{n}^{T}$ for all $n \neq 2$ are smooth functions.

Proof. From Proposition 4.1 and Eq. (1) we see that $\left(x_{n}, k_{n} ; \ldots ; x_{1}, k_{1}\right) \in W F\left(\omega_{n}^{T}\right)$ implies $\left(x_{1}, k_{1}\right) \in V^{*+} \cup \mathcal{Z}$ and $\left(x_{n}, k_{n}\right) \in V^{*-} \cup \mathcal{Z}$. However, because $\omega$ is a generalised free field state all truncated $n$-point distributions with $n \neq 2$ are symmetric by Proposition 3.4. This means that each $\left(x_{i}, k_{i}\right)$ must be in $\left(V^{*+} \cup \mathcal{Z}\right) \cap\left(V^{*-} \cup \mathcal{Z}\right)=\mathcal{Z}$, i.e. $k_{i}=0$. It follows that $W F\left(\omega_{n}^{T}\right)=\emptyset$ and hence $\omega_{n}^{T}$ is smooth for $n \neq 2$. The result for $n=2$ follows from $\omega_{2}-\omega_{2}^{T}=\omega_{1} \otimes \omega_{1}$.

Corollary 4.3. Let $\omega$ be a generalised Hadamard state which is also a generalised free field state. Then $\omega$ satisfies the $\mu S C$ with smooth, resp. causal, resp. light-like immersions if $W F\left(\omega_{2-}\right) \subset \Gamma_{2}^{\bullet}$, where $\bullet$ denotes no superscript, resp. c, resp. ll. More precisely, for each point in $W F\left(\omega_{n}\right)$ we can find an instantiating graph $G \in \mathcal{G}_{n}$ which is a disconnected union of graphs in $\mathcal{G}_{2}$ that instantiate points in $W F\left(\omega_{2}\right)=W F(E) \cap \Gamma_{2}$.

Proof. This follows immediately from Theorem 4.2, Eq. (1) and the properties of the cones $\Gamma_{n}^{\bullet}$ in Proposition 2.5.

The singularity structure that we derived in Theorem 4.2 and Corollary 4.3 is what one would expect of quasi-free states, because of Eq. (1) (see [2]). It is nice to see that this form persists when the state is only required to satisfy scalar commutation relations. Analogous results also hold in the analytic case, for vector-valued fields and in the case of anti-commutation relations.

[2] describes a point in $T^{*} M^{5} \backslash \mathcal{Z}$ that is not in $\Gamma_{5}^{c}$ and one wonders whether such a point can be in the wave front set of the 5-point distribution of a state. We have just 
proved that for generalised free fields this possibility is excluded. Moreover, our result also implies that the $\mu \mathrm{SC}$ with light-like curves includes more than just free fields and their Wick powers [2], namely generalised free fields with any suitable commutator function. (We will leave the existence of a sufficiently large class of such fields in curved spacetime for a future publication.)

An easy consequence of the analytic case of Theorem 4.2 is the following characterisation of generalised free field states:

Proposition 4.4. Let $\omega$ be a causal state satisfying the A $\mu S C$. Then $\omega$ is a generalised free field state if and only if $\omega_{n}^{T}$ is analytic for all $n \neq 2$.

Proof. If $\omega$ is a generalised free field state the conclusion follows from the analytic version of Theorem 4.2. For the converse we use causality to prove by induction on $n$ that every $\omega_{n}^{T}$ is symmetric when all arguments are space-like separated. Analytic continuation for $n \neq 2$ then proves their symmetry everywhere and we may then apply Proposition 3.4.

As another easy result we show that the class of generalised Hadamard states of a generalised free field is closed under operations:

Proposition 4.5. Let $\omega$ be a generalised Hadamard and generalised free field state on $\mathcal{U}_{M}$ and let $A \in \mathcal{U}_{M}$ be any operator such that $\omega\left(A^{*} A\right)=1$. Then the state $\omega^{A}$, defined by $\omega^{A}(B):=\omega\left(A^{*} B A\right)$, is a generalised Hadamard and generalised free field state on $\mathcal{U}_{M}$.

Notice that for given $A$ the expression $\omega\left(A^{*} B A\right)$ may involve arbitrary high $n$-point distributions, depending on the choice of $B$, so without an estimate on the wave front sets of higher $n$-point distributions this result sounds rather surprising.

Proof. We may write $A=\sum_{i=1}^{n} f_{i}^{(i)} \otimes \cdots \otimes f_{1}^{(i)}$ for some $n$ and $f_{j}^{(i)} \in C_{0}^{\infty}(M)$. The two-point distribution of $\omega^{A}$ is then a sum of terms of the form

$$
\omega_{i+k+2}\left(\overline{f_{1}^{(i)}}, \ldots, \overline{f_{i}^{(i)}}, x_{2}, x_{1}, f_{k}^{(k)}, \ldots, f_{1}^{(k)}\right)
$$

which are distributions in $x_{1}, x_{2}$. The wave front set of each such term can be estimated using standard arguments (see [9] Theorem 8.2.12) as a subset of

$$
\left\{\left(x_{2}, k_{2} ; x_{1}, k_{1}\right) \mid\left(y_{1}, 0 ; \ldots ; y_{i}, 0 ; x_{2}, k_{2} ; x_{1}, k_{1} ; z_{k}, 0 ; \ldots ; z_{1}, 0\right) \in W F\left(\omega_{i+k+2}\right)\right\}
$$

which is a subset of $\Gamma_{2}$. The wave front set of a sum of such terms is also contained in $\Gamma_{2}$ by Proposition 2.5 and therefore $\omega^{A}$ is a generalised Hadamard state. That it is a generalised free field state follows from Eq. (6).

To close this section we prove the following lemma on the comparisons of the $n$-point distributions of two states, generalising Lemma 2.9.

Lemma 4.6. Consider two generalised Hadamard states $\omega, \omega^{\prime}$, which both satisfy commutation relations with the same commutator $E$ such that $W F(E) \neq \emptyset$. For any $n \geq 0$ we have that $\omega_{n+2}-\omega_{n+2}^{\prime}$ is smooth if and only if $\omega_{n} \equiv \omega_{n}^{\prime}$. 
Proof. The case $n=0$ follows from Lemma 2.9. For $n \geq 1$ we first suppose that $\omega_{n} \equiv \omega_{n}^{\prime}$. For any index $1 \leq i<n$ we then have $\left(\omega_{n+2}-\omega_{n+2}^{\prime}\right)\left(x_{n+2}, \ldots, x_{1}\right)=\left(\omega_{n+2}-\right.$ $\left.\omega_{n+2}^{\prime}\right)\left(x_{n+2}, \ldots, x_{i}, x_{i+1}, \ldots, x_{1}\right)$, where we swapped the indices $i$ and $i+1$ and the commutator terms vanish by the assumption. We can therefore permute indices ad lib. and in this way we derive $\left(\omega_{n+2}-\omega_{n+2}^{\prime}\right)\left(x_{n+2}, \ldots, x_{1}\right)=\left(\omega_{n+2}-\omega_{n+2}^{\prime}\right)\left(x_{1}, \ldots, x_{n+2}\right)$. Using the assumption that both states are generalised Hadamard states and Items two and four of Proposition 2.5 we find that $W F\left(\omega_{n+2}-\omega_{n+2}^{\prime}\right) \subset \Gamma_{n+2} \cap-\Gamma_{n+2}=\emptyset$. This proves that $\omega_{n+2}-\omega_{n+2}^{\prime}$ is smooth.

For the opposite direction we assume that $\omega_{n+2}-\omega_{n+2}^{\prime}$ is smooth and we let the symbol $\sim$ denote equality modulo terms $w$ such that $W F(w) \cap T^{*} M \times V^{*+} \times T^{*} M \times \cdots \times$ $T^{*} M=\emptyset$, i.e. we are interested in the direction of the covectors in the $n+1^{\text {st }}$ slot (from the right). Using the expressions for $\omega_{n+2}$ and $\omega_{n+2}^{\prime}$ in terms of truncated $n$-point distributions (1) we compute:

$$
\begin{aligned}
0 & \sim \omega_{n+2}-\omega_{n+2}^{\prime} \sim \omega_{2} \otimes \omega_{n}-\omega_{2}^{\prime} \otimes \omega_{n}^{\prime} \\
& \sim \omega_{2} \otimes \omega_{n}-\omega_{2} \otimes \omega_{n}^{\prime}=\omega_{2} \otimes\left(\omega_{n}-\omega_{n}^{\prime}\right),
\end{aligned}
$$

where we used the result for $n=0$ to get to the last line. If $w_{n}:=\omega_{n}-\omega_{n}^{\prime} \neq 0$ is not identically 0 then we can find test-functions $f_{1}, \ldots, f_{n}$ such that $c:=w_{n}\left(f_{n}, \ldots, f_{1}\right) \neq 0$, which leads to a contradiction as follows. Notice that

$$
\begin{aligned}
W F\left(\omega_{2}\right)= & W F\left(c \cdot \omega_{2}\right)=W F\left(\omega_{2} \cdot w_{n}\left(f_{n}, \ldots, f_{1}\right)\right) \\
\subset & \left\{\left(x_{n+2}, k_{n+2} ; x_{n+1}, k_{n+1}\right) \mid \text { for some } x_{i} \in \operatorname{supp}\left(f_{i}\right), i=1, \ldots, n\right. \\
& \left.\left(x_{n+2}, k_{n+2} ; x_{n+1}, k_{n+1} ; x_{n}, 0 ; \ldots ; x_{1}, 0\right) \in W F\left(\omega_{2} \otimes w_{n}\right)\right\},
\end{aligned}
$$

by Theorem 8.2.12 of [9]. Because $\omega_{2} \otimes w_{n} \sim 0$ and because $\omega_{2}$ is a generalised Hadamard state we find that $W F\left(\omega_{2}\right)=\emptyset$. However, by Proposition 3.2 this implies that $W F(E) \cap \Gamma_{2}=\emptyset$ and hence $W F(E) \cap-\Gamma_{2}=\emptyset$ and $W F(E)=\emptyset$. This contradicts the assumption on $E$, so we must have $w_{n} \equiv 0$.

The same statement still holds when the commutators $E$ and $E^{\prime}$ of the two states differ by a smooth function.

\section{Two Theorems Generalised to Curved Spacetimes}

We now discuss the generalisation of two theorems from Wightman field theory to curved spacetimes, illustrating the strength and the limitations of the commutation relations in that setting. First we generalise a result due to Borchers and Zimmermann [1] concerning the self-adjointness of field operators. Then we consider the generalisation of (a weak form of) the Jost-Schroer theorem.

The result of [1] gives a sufficient condition for the symmetric operator $\Phi^{\omega}(f)$ with a given $f \in C_{0}^{\infty}(M, \mathbb{R})$ to be self-adjoint. To discuss its generalisation we recall the following notion:

Definition 5.1. A vector $\psi$ in a Hilbert space $\mathcal{H}$ is an analytic vector for a (possibly unbounded) linear operator $T$ on $\mathcal{H}$ iff the series $\sum_{n=0}^{\infty} \frac{\left\|T^{n} \psi\right\|}{n !} z^{n}$ has a non-zero radius of convergence. (In particular we require that $\psi$ is in the domain of each $T^{n}$.)

Notice that for a bounded linear operator $T$ all vectors are analytic. The following elementary lemma is adapted from [1]: 
Lemma 5.2. For a vector $\psi$ in the Hilbert space $\mathcal{H}$ and a symmetric linear operator $T$ on $\mathcal{H}$ the following are equivalent:

1. $\psi$ is analytic for $T$,

2. there is a constant $c>0$ such that $\left\|T^{n} \psi\right\| \leq n ! c^{n}$,

3. $\sum_{n=0}^{\infty} \frac{\left|\left\langle\psi, T^{n} \psi\right\rangle\right|}{n !} z^{n}$ has a non-zero radius of convergence,

4. there is a constant $c>0$ such that $\left|\left\langle\psi, T^{n} \psi\right\rangle\right| \leq n ! c^{n}$.

Proof. See loc. cit. Sect. 2.

For a Wightman field theory in Minkowski spacetime Borchers and Zimmermann [1] used causality and the Reeh-Schlieder theorem to prove that a field operator $\Phi^{\omega}(f)$ is self-adjoint as soon as the vacuum vector $\Omega_{\omega}$ is analytic. An analogous proof can be given in curved spacetime, whenever the state $\omega$ is causal and has the Reeh-Schlieder property, i.e. the GNS-vector $\Omega_{\omega}$ is cyclic for all local algebras. The latter can be ensured e.g. by imposing the $\mathrm{A} \mu \mathrm{SC}$ (see $[17,21]$ ), but unfortunately it is not clear whether all analytic spacetimes admit states satisfying the A $\mu \mathrm{SC}$, or whether all (smooth) spacetimes have states with the Reeh-Schlieder property. We now prove that the conclusion of Borchers and Zimmermann can also be obtained without recourse to the Reeh-Schlieder theorem if we assume that the state is a generalised free field state. For the proof we adapt an idea of Nelson [14].

Theorem 5.3. If $\omega$ is a generalised free field state on $\mathcal{U}_{M}$ with some commutator $E$ and $\Omega_{\omega}$ is an analytic vector for $\Phi^{\omega}(f)$ for some $f \in C_{0}^{\infty}(M, \mathbb{R})$, then all vectors $\pi_{\omega}(A) \Omega_{\omega}$ with $A \in \mathcal{U}_{M}$ are analytic vectors for $\Phi^{\omega}(f)$ and this operator is essentially self-adjoint.

Proof. First assume that $\psi \in \pi_{\omega}\left(\mathcal{U}_{M}\right) \Omega_{\omega}$ is an analytic vector for $\Phi^{\omega}(f)$ for given $f \in C_{0}^{\infty}(M, \mathbb{R})$. For any $h \in C_{0}^{\infty}(M)$ we will prove that $\Phi^{\omega}(h) \psi$ is an analytic vector for $\Phi^{\omega}(f)$. To see this we note that for $n \geq 1$ we have

$$
\Phi(f)^{n} \Phi(h)=\Phi(h) \Phi(f)^{n}+n i E(f, h) \Phi(f)^{n-1},
$$

which may easily be proved by induction. Using this we compute:

$$
\begin{aligned}
\left|\left\langle\Phi^{\omega}(h) \psi, \Phi^{\omega}(f)^{n} \Phi^{\omega}(h) \psi\right\rangle\right| \leq & \left|\left\langle\Phi^{\omega}(\bar{h}) \Phi^{\omega}(h) \psi, \Phi^{\omega}(f)^{n} \psi\right\rangle\right| \\
& +n|E(F, h)| \cdot\left|\left\langle\Phi^{\omega}(h) \psi, \Phi^{\omega}(f)^{n-1} \psi\right\rangle\right| \\
\leq & c\left\|\Phi^{\omega}(f)^{n} \psi\right\|+c n\left\|\Phi^{\omega}(f)^{n-1} \psi\right\|,
\end{aligned}
$$

where the constant $c>0$ may depend on $f$ and $h$, but not on $n$. The assumption that $\psi$ is analytic then implies that (see Lemma 5.2)

$$
\left|\left\langle\Phi^{\omega}(h) \psi, \Phi^{\omega}(f)^{n} \Phi^{\omega}(h) \psi\right\rangle\right| \leq c\left(c^{\prime}\right)^{n} n !+c n\left(c^{\prime}\right)^{n-1}(n-1) ! \leq C^{n} n !
$$

for suitable constants $c^{\prime}, C>0$. By Lemma 5.2 this implies that $\Phi^{\omega}(h) \psi$ is an analytic vector for $\Phi^{\omega}(f)$.

Now assume that $\Omega_{\omega}$ is an analytic vector for $\Phi^{\omega}(f)$. We can then repeatedly apply the result of the previous paragraph to prove that any vector of the form $\Phi^{\omega}\left(h_{m}\right) \cdots \Phi^{\omega}\left(h_{1}\right)$ $\Omega_{\omega}$ is an analytic vector. Because the set of analytic vectors for a given operator is a linear space, every vector in $\pi_{\omega}\left(\mathcal{U}_{M}\right) \Omega_{\omega}$ is analytic. This provides a dense set of analytic vectors, so we can apply Nelson's theorem ([14] Lemma 5.1) to conclude that $\Phi^{\omega}(f)$ is essentially self-adjoint. 
The analyticity of $\Omega_{\omega}$ can be formulated conveniently in terms of the $n$-point distributions by Lemma 5.2 and in terms of the truncated $n$-point distributions too (for a proof we refer to [1]):

Proposition 5.4. $\Omega_{\omega}$ is an analytic vector for $\Phi^{\omega}(f)$ if and only if there is a $d>0$ such that $\left|\omega_{n}^{T}\left(f^{\otimes n}\right)\right|<n ! d^{n}$ for all $n \in \mathbb{N}$.

The condition of the previous theorem may not always be satisfied, as we will now illustrate with the following

Example. In Minkowski spacetime we will construct a translation invariant free field state $\tilde{\omega}$ which satisfies the $\mathrm{A} \mu \mathrm{SC}$, but whose $G N S$-vector $\Omega_{\tilde{\omega}}$ is not analytic for any non-zero smeared field operator $\Phi^{\tilde{\omega}}(f), f \in C_{0}^{\infty}\left(M_{0}, \mathbb{R}\right)$. (We will not discuss the question whether these operators are essentially self-adjoint.)

Let $\omega$ denote the Minkowski vacuum state with two-point distribution $\omega_{2}$. We set $w_{2}\left(x_{2}, x_{1}\right):=\int e^{-i k \cdot\left(x_{1}-x_{2}\right)} e^{-k_{0}^{2}} \delta\left(k^{2}-m^{2}\right) d k$, which is an analytic, real-valued, symmetric and translation invariant bi-solution of the Klein-Gordon equation of positive type. Next we define the two-point distributions $\omega_{2}^{j}:=e^{j} w_{2}+\omega_{2}$ for each $j \in \mathbb{N}$ and we note that the anti-symmetric part is $\omega_{2-}^{j}=\omega_{2-}$. Each $\omega_{2}^{j}$ defines a quasi-free state $\omega^{j}$ on the Weyl-algebra (see [12]) and hence also on the Borchers-Uhlmann algebra, because a quasi-free state is regular (cf. Proposition 5.4). Each of the states $\omega^{j}$ is a translation invariant, Hadamard, free field state satisfying the A $\mu$ SC. (Note however that they are not Lorentz-invariant, because $w_{2}$ is not Lorentz invariant.)

Now we define the state $\tilde{\omega}$ by $\tilde{\omega}:=e^{-1} \sum_{j=0}^{\infty} \frac{1}{j !} \omega^{j}$. Note that $\tilde{\omega}\left(A^{*} A\right) \geq 0$ and $\tilde{\omega}_{0}(I)=1$, so it is indeed a state. It follows from the properties of the $\omega^{j}$ that $\tilde{\omega}$ is translation invariant and that it is a free field state. To see that $\tilde{\omega}$ is continuous we note that $\tilde{\omega}_{2 n-1}=0$ for $n \in \mathbb{N}$ and that for all $n, N \in \mathbb{N}$ :

$$
\begin{aligned}
& e^{-1} \sum_{j=0}^{N} \frac{1}{j !} \omega_{2 n}^{j}=e^{-1} \sum_{P \in \mathcal{P}_{n}} \sum_{j=0}^{N} \frac{1}{j !}\left(e^{j} w_{2}+\omega_{2}\right)^{\otimes n} \circ \pi_{P} \\
& =e^{-1} \sum_{P \in \mathcal{P}_{n}} \sum_{k=0}^{n} \sum_{j=0}^{N} \frac{e^{k j}}{j !}\left(w_{2}^{\otimes k} \otimes \omega_{2}^{\otimes(n-k)}+\cdots+\omega_{2}^{\otimes(n-k)} \otimes w_{2}^{\otimes(n-k)}\right) \circ \pi_{P},
\end{aligned}
$$

where the operation $\pi_{P}$ denotes the permutation that corresponds to the partition $P$ of the set $\{1, \ldots, n\}$ (see Eq. (1) and Definition 2.2) and the dots in the last line indicate all the different orderings of the factors $w_{2}$ and $\omega_{2}$. Taking the limit we see that the sum over $j$ converges so that

$$
\tilde{\omega}_{2 n}=\sum_{P \in \mathcal{P}_{n}} \sum_{k=0}^{n} e^{e^{k}-1}\left(w_{2}^{\otimes k} \otimes \omega_{2}^{\otimes(n-k)}+\cdots+\omega_{2}^{\otimes(n-k)} \otimes w_{2}^{\otimes(n-k)}\right) \circ \pi_{P},
$$

which exhibits $\tilde{\omega}_{2 n}$ as a finite sum of distributions. It also follows from Eq. (7) that $\tilde{\omega}$ satisfies the $\mathrm{A} \mu \mathrm{SC}$.

Finally we prove that $\Omega_{\tilde{\omega}}$ is not an analytic vector for any non-zero $\Phi^{\tilde{\omega}}(f)$ with $f \in C_{0}^{\infty}\left(M_{0}, \mathbb{R}\right)$. Suppose that $\Omega_{\tilde{\omega}}$ is an analytic vector for a given $\Phi^{\tilde{\omega}}(f)$. By Lemma 5.2 there is a constant $c>0$ such that

$$
c^{2 n}(2 n) ! \geq \tilde{\omega}_{2 n}\left(f^{\otimes 2 n}\right) \geq \frac{(2 n) !}{2^{n} n !} e^{e^{n}-1} w_{2}(f, f)^{n},
$$


where we used Eq. (7) and the positive type of $\omega_{2}$ and $w_{2}$ for the last inequality. Using $\ln n \leq n$ we find $n ! \leq n^{n} \leq e^{n^{2}}$ and hence

$$
c^{2 n} \geq\left(\frac{w_{2}(f, f)}{2}\right)^{n} e^{n^{3} / 6-n^{2}} .
$$

If $w_{2}(f, f) \neq 0$ we can take logarithms on both sides and let $n \rightarrow \infty$ to find a contradiction. If $w_{2}(f, f)=0$, on the other hand, we use the positivity and the support of $\hat{w}_{2}$ to deduce that $\omega_{2}(f, f)=0$ too and hence $\tilde{\omega}_{2}(f, f)=0$. This means that $\Phi^{\tilde{\omega}}(f)$ annihilates $\Omega_{\tilde{\omega}}$ and it commutes with all other smeared field operators, so that $\Phi^{\tilde{\omega}}(f)=0$ (cf. the proof of Proposition 5.5 below).

Now we turn to an analogue of the Jost-Schroer theorem (see $[4,11,15])$, which provides a way to recognise free field states. In the Wightman framework this theorem says that any state whose two-point distribution is that of a free field must be a free field state $^{3}$. (Recall that this means it satisfies the Klein-Gordon equation and the canonical commutation relations.)

As before we can prove our result by using commutation relations to replace the analyticity that is due to the spectrum condition of the Wightman axioms. Note, however, that this makes part of the result, namely the proof of the commutation relations, trivial. The following is therefore a generalisation of a very weak form of the Jost-Schroer theorem:

Proposition 5.5. Let $\omega$ be a generalised free field state and assume that $\omega_{2}$ is the twopoint distribution of a free-field state, i.e. it satisfies the Klein-Gordon equation for some mass $m$ and scalar curvature coupling $\xi$ and $\omega_{2-}=\frac{i}{2} E_{m, \xi}$. Then $\omega$ is a free field state.

(The same result also works for other linear partial differential operators.)

Proof. Let $K$ denote the Klein-Gordon operator with mass $m$ and coupling $\xi$. For any $f \in C_{0}^{\infty}(M)$ we have $K \Phi(f)=\Phi(K f)$, because the Klein-Gordon operator is formally self-adjoint. This implies that

$$
\left|\omega_{n}\left(f_{n}, \ldots, f_{2}, K f_{1}\right)\right| \leq\left\|\Phi^{\omega}\left(\bar{f}_{2}\right) \cdots \Phi^{\omega}\left(\bar{f}_{n}\right) \Omega_{\omega}\right\| \cdot\left\|\Phi^{\omega}\left(K f_{1}\right) \Omega_{\omega}\right\|=0,
$$

because $\left\|\Phi^{\omega}\left(K f_{1}\right) \Omega_{\omega}\right\|^{2}=\omega_{2}\left(K \bar{f}_{1}, K f_{1}\right)=0$. Therefore every $\omega_{n}$ satisfies the KleinGordon equation in the first (rightmost) argument. One proves by induction that the same is then true for $\omega_{n}^{T}$, using Eq. (1). For a generalised free field state we can then apply Proposition 3.4 and find that $\omega_{n}^{T}$ satisfies the Klein-Gordon equation in all arguments for $n \neq 2$. For $n=2$ this is true by the assumption on $\omega_{2}$. Using Eq. (1) once more shows that the $\omega_{n}$ satisfy the Klein-Gordon equation in all arguments, which completes the proof.

3 A related result, due to Greenberg [5], says that a state must be a generalised free field state if the Källen-Lehmann representation of the two-point distribution

$$
\omega_{2-}\left(x_{2}, x_{1}\right)=\int \rho_{K L}\left(m^{2}\right) \omega_{2-}^{m}\left(x_{2}, x_{1}\right) d m^{2}
$$

in terms of the free field commutator functions of mass $m, \omega_{2-}^{m}$, has a positive measure $\rho_{K L}$ whose support satisfies certain restrictions. In the Wightman framework every $\omega_{2}$ - allows a Källen-Lehmann representation, but in curved spacetime such a tool is not available, so at present it makes no sense to consider the generalisation of this result. Moreover, our current strategy of weakening the Wightman axioms and assuming commutation relations instead would render the statement trivial. 
Alternatively we could drop the assumption that $\omega$ is a generalised free field state and require causality and the $\mathrm{A} \mu \mathrm{SC}$ (or the Reeh-Schlieder property) instead. This certainly allows us to prove that $\omega$ satisfies the Klein-Gordon equation as follows:

Proposition 5.6. Let $\omega$ be a causal state satisfying the A $\mu S C$. If $K_{x} \omega_{2}(x, y)=0$ then $\omega$ satisfies the Klein-Gordon equation.

Proof. By A $\mu \mathrm{SC}, \omega$ has the Reeh-Schlieder property, i.e. $\Omega_{\omega}$ is a cyclic vector for every local algebra [21]. Now $\Phi^{\omega}(K f)$ annihilates $\Omega_{\omega}$ for every $f \in C_{0}^{\infty}(M)$ and $\Phi^{\omega}(K f) \cdot \pi_{\omega}(B) \Omega_{\omega}=0$ for any $B$ that commutes with $\Phi(f)$. By causality and the Reeh-Schlieder property we conclude that $\Phi^{\omega}(K f)$ annihilates a dense set of vectors and hence $\Phi^{\omega}(K f)=0$ (because the operator is closable).

Note, however, that it is not at all clear whether the state also satisfies the canonical commutation relations. The proof of [15], e.g., uses Poincaré invariance, the full strength of the spectrum condition and the uniqueness of the vacuum ${ }^{4}$. We will not investigate what other assumptions are necessary to recover the strong version of the Jost-Schroer theorem, but for completeness we do provide the following:

Example. We construct a state satisfying the assumptions of Proposition 5.6 with the canonical commutator function, but which is not a generalised free field state. For this purpose we let $\omega^{1}$ denote the quasi-free state on Minkowski spacetime with two-point distribution $\omega_{2}^{1}=2 \omega_{2}^{0}$, where $\omega^{0}$ is the Minkowski vacuum. We let $\omega^{2}$ be the state with $\omega_{n}^{2}=0$ for all $n>0$ and we note that the mixed state $\omega^{3}:=\frac{1}{2}\left(\omega^{1}+\omega^{2}\right)$ serves our purpose by considering the four-point distribution:

$$
\omega_{4}^{3}\left(x_{4}, x_{3}, x_{2}, x_{1}\right)-\omega_{4}^{3}\left(x_{3}, x_{4}, x_{2}, x_{1}\right)=2 i \omega_{2-}^{3}\left(x_{4}, x_{3}\right) \omega_{2}^{3}\left(x_{2}, x_{1}\right) .
$$

\section{Applications and Outlook}

[13] already mentions the class of Hadamard states whose truncated $n$-point distributions are smooth functions for all $n \neq 2$ as an interesting class. Later [7,8] discuss perturbation theory by constructing an extended *-algebra of Wick powers and timeordered products of a free field and find that the continuous states on this algebra are exactly the Hadamard states of this class. Our Theorem 4.2 shows that the condition on the truncated $n$-point distributions is automatically satisfied for (generalised) free fields due to the scalar commutation relations, so the class of all Hadamard states is the class of interest for perturbative quantum field theory. Furthermore, Corollary 4.3 shows that for a generalised free field any generalised Hadamard state satisfies the $\mu \mathrm{SC}$ and Proposition 4.5 tells us that the class of generalised Hadamard states is closed under operations, which is useful to know from a fundamental point of view. Our Theorem 4.2 and Corollary 4.3 could find further applications in perturbative quantum field theory around a generalised free field, rather than around a free field. Such an approach has been suggested in [3] as a way to gain insight in the AdS-CFT correspondence.

Concerning the strength of the assumption that a state is a generalised free field state we have discussed the generalisation of two results from the Wightman framework to curved spacetimes. We showed that in some circumstances our assumption can replace

\footnotetext{
4 In this connection it should also be noted that generalised free fields need not have the time-slice property, so then the commutation relations cannot be proved in curved spacetime via a spacetime-deformation argument as in [22].
} 
the existing arguments based on analyticity, as in Theorem 5.3 that generalised a result of Borchers and Zimmerman. For the Jost-Schroer theorem the situation was more delicate: a weak form of this theorem can be proved in curved spacetimes by assuming that a state is a generalised free field state. However, it is not known if one can prove that a state is a (generalised) free field under suitable circumstances without assuming commutation relations in the first place.

Finally we note that the proofs we used were all elementary applications of the calculus of wave front sets of (Hilbert space-valued) distributions and the combinatorics of (truncated) $n$-point distributions. Both can be generalised to vector-valued fields and to anti-commutation relations in a straightforward manner (see e.g. [18] Prop. 4.2.17 for the result that a Hadamard state of the free Dirac field satisfies the $\mu \mathrm{SC}$ ).

Note added in proof. Our Proposition 3.4 was already known as Lemma 5.2 of Gottschalk and Thaler, "An indefinite metric model for interacting quantum fields on globally hyperbolic space-times", Ann. Henri Poincare 4 (2003) 637-659. We thank Thomas-Paul Hack for bringing this to our attention.

Acknowledgements. I would like to thank Chris Fewster, Bernard Kay, Karl-Henning Rehren and Pedro Lauridsen Ribeiro for helpful suggestions and discussions. This research was supported by the German Research Foundation (Deutsche Forschungsgemeinschaft (DFG)) through the Institutional Strategy of the University of Göttingen and the Graduiertenkolleg 1493 "Mathematische Strukturen in der modernen Quantenphysik". The results of Sect. 4 were obtained during the preparation of my $\mathrm{PhD}$ thesis at the University of York.

Open Access This article is distributed under the terms of the Creative Commons Attribution Noncommercial License which permits any noncommercial use, distribution, and reproduction in any medium, provided the original author(s) and source are credited.

\section{References}

1. Borchers, H.-J., Zimmermann, W.: On the self-adjointness of field operators. Nuovo Cimento 31(10), 1047-1059 (1964)

2. Brunetti, R., Fredenhagen, K., Köhler, M.: The microlocal spectrum condition and Wick polynomials of free fields on curved spacetimes. Commun. Math. Phys. 180, 633-652 (1996)

3. Dütsch, M., Rehren, K.-H.: Generalized free fields and the AdS-CFT correspondence. Ann. Henri Poincaré 4, 613-635 (2003)

4. Federbush, P.G., Johnson, K.A.: Uniqueness property of the two-fold vacuum expectation value. Phys. Rev. 120, 1926 (1960)

5. Greenberg, O.W.: Heisenberg fields which vanish on domains of momentum space. J. Math. Phys. 3, 859866 (1962)

6. Hall, D., Wightman, A.S.: A theorem on invariant analytic functions with applications to relativistic quantum field theory. Mat.-Fys. Medd. Danske Vid. Selsk. 31, 1-41 (1957)

7. Hollands, S., Wald, R.M.: Local Wick polynomials and time ordered products of quantum fields in curved spacetime. Commun. Math. Phys. 223, 289-326 (2001)

8. Hollands, S., Ruan, W.: The state space of perturbative quantum field theory in curved spacetimes. Ann. Henri Poincaré 3, 635-657 (2002)

9. Hörmander, L.: The analysis of linear partial differential operators I. Berlin: Springer, 2003

10. Jost, R.: The general theory of quantized fields. Providence, RI: Amer. Math. Soc., 1965

11. Jost, R.: Properties of Wightman functions. In: Lectures on field theory and the many-body problem, New York: Academic Press, 1961, pp. 127-145

12. Kärsten, F.: Klassifikation der unitär invarianten regulären Zustände der Weylalgebra der CCR über einem separablen Hilbertraum. Report Math 06, 1989

13. Kay, B.S.: Quantum field theory in curved spacetimes. In: Mathematical physics X (Proceedings, Leipzig, Germany 1991), K. Schmüdgen (ed.), Berlin: Springer, 1992

14. Nelson, E.: Analytic Vectors. Ann. of Math. 70, 572-615 (1959)

15. Pohlmeyer, K.: The Jost-Schroer theorem for zero-mass fields. Commun. Math. Phys. 12, 204-211 (1969)

16. Radzikowski, M.J.: Micro-Local Approach to the Hadamard Condition in Quantum Field Theory on Curved Space-Time. Commun. Math. Phys. 179, 529-553 (1996)

17. Sanders, K.: On the Reeh-Schlieder property in curved spacetime. Commun. Math. Phys. 288, 271-285 (2009) 
18. Sanders, K.: Aspects of locally covariant quantum field theory. $\mathrm{PhD}$ thesis, York (July 2008), also available at http://arXiv.org/abs/0809.4828v1[math-ph], 2008

19. Schaefer, H.H.: Topological vector spaces. New York: Macmillan, 1966

20. Schmüdgen, K.: Unbounded operator algebras and representation theory. Basel: Birkhäuser Verlag, 1990

21. Strohmaier, A., Verch, R., Wollenberg, M.: Microlocal analysis of quantum fields on curved space-times: analytic wavefront sets and Reeh-Schlieder theorems. J. Math. Phys. 43, 5514-5530 (2002)

22. Verch, R.: A spin-statistics theorem for quantum fields on curved spacetime manifolds in a generally covariant framework. Commun. Math. Phys. 223, 261-288 (2001)

23. Wald, R.M.: Quantum field theory in curved spacetime and black hole thermodynamics. ChicagoLondon: The University of Chicago Press, 1994

Communicated by Y. Kawahigashi 